SCIENTIFIC REPORT

\title{
Suprachoroidal haemorrhage complicating cataract surgery in the UK: a case control study of risk factors
}

\author{
R Ling, S Kamalarajah, M Cole, C James, S Shaw
}

Br J Ophthalmol 2004;88:474-477. doi: 10.1136/bjo.2003.026179

Aims: To study the risk factors for suprachoroidal haemorrhage $(\mathrm{SCH})$ complicating cataract surgery in the United Kingdom.

Methods: 109 cases of SCH complicating cataract surgery prospectively collected through the British Ophthalmological Surveillance Unit were compared with 449 controls that underwent cataract extraction from 13 "control centres" throughout UK in a case control study. 40 systemic, ophthalmic, and operative variables were examined.

Results: Statistically significant risk factors for $\mathrm{SCH}$ in univariate analysis included: older age $(p<0.001)$, taking at least one cardiovascular medication $(p<0.001)$, peripheral vascular disease $(p=0.014)$, hyperlipidaemia $(p=0.005)$, glaucoma $(p<0.001)$, elevated preoperative intraocular pressure $(p<0.001)$, sub-Tenon's local anaesthesia (LA) $(p<0.001)$, topical LA $(p<0.001)$, the lack of orbital compression following LA $(p<0.001)$, posterior capsule (PC) rupture before $\mathrm{SCH}(p<0.001)$, elective extracapsular cataract extraction (ECCE) $(p=0.038)$, and phacoemulsification conversion $(p<0.001)$. Logistic regression analysis identified the following significant independent risk factors: older age, taking at least one cardiovascular medication, glaucoma, elevated preoperative intraocular pressure, the lack of orbital compression, PC rupture before $\mathrm{SCH}$, elective ECCE, and phacoemulsification conversion. Conclusion: The results allow identification of patients at risk of $\mathrm{SCH}$. Attention to the various modifiable preoperative and intraoperative risk factors is recommended in order to minimise the risk of $\mathrm{SCH}$.

S uprachoroidal haemorrhage ( $\mathrm{SCH}$ ) is a rare but potentially devastating complication of cataract surgery. Information on the risk factors for $\mathrm{SCH}$ is often extrapolated from case series of SCH from a wide variety of procedures, ${ }^{12}$ or from small cataract surgery series, ${ }^{3}{ }^{4}$ mostly conducted at the time of extracapsular cataract extraction (ECCE).

The present study was undertaken to examine the risk factors for SCH in modern cataract surgery by using the epidemiological methodology of a case control study.

\section{MATERIALS AND METHODS \\ Cases}

Information on 109 new cases of SCH complicating cataract surgery were prospectively collected through the surveillance scheme of the British Ophthalmological Surveillance Unit $(B O S U)^{5}$ from November 2000 to October 2001. Case definition was haemorrhage in the suprachoroidal space during cataract surgery, diagnosed by the surgeon, excluding cases that combine cataract extraction with another intraocular procedure. Detailed information was obtained from the reporting ophthalmologist using a standard questionnaire.

\section{Controls}

Information on 449 controls was prospectively collected during the study period, using a standard format developed simultaneously as the questionnaire for SCH cases. "Control centres" were selected by a clustered, stratified, random sampling procedure by dividing the UK into nine regions. Within each region, centres were chosen by applying computer generated random numbers to a list of hospitals with the probability of selection proportional to the consultant number. Thirteen of 20 selected centres participated, each providing approximately 40 controls systematically sampled from a chronological list of patients who underwent cataract surgery between March and August 2000. This ensured there was a minimum postoperative interval of 6 months at the time of data sampling (March to July 2001).

Control data were shared with the BOSU study of "endophthalmitis following cataract surgery", and simultaneously sampled by two study coordinators (RL, six centres; SK eight centres). To ensure standardisation, data from one centre were independently sampled at the beginning of the study and found to have $98.5 \%$ concordance between the coordinators.

\section{Risk factors examined}

The following variables were studied in both the cases and controls:

Systemic. Age, sex, diabetes, HbAlC and blood glucose (if diabetic), hypertension, preoperative blood pressure (BP), heart failure, ischaemic heart disease, cerebral vascular accident, peripheral vascular disease, hyperlipidaemia, respiratory disease, bleeding diathesis, anaemia, medications (specifically cardiovascular medications, aspirin and warfarin).

Ophthalmic. Glaucoma, ocular comorbidity (specifically uveitis, past trauma), previous intraocular procedure, axial length, intraocular pressure (IOP) prior to cataract surgery, and preoperative visual acuity.

Operative. Eye (right/left), surgeon grade, anaesthetist grade, type of anaesthesia, anaesthetic complications, orbital compression following local anaesthesia (LA), peak intraoperative pulse and BP, operative procedure (phacoemulsification, elective ECCE, or phacoemulsification conversion), section (corneal/scleral), posterior capsule (PC) rupture, and the timing of PC rupture in relation to $\mathrm{SCH}$ in the cases.

Posterior capsule was ruptured in 57 (52.3\%) cases. Thirty five occurred during or after SCH and can be regarded as an

Abbreviations: BP, blood pressure; BOSU, British Ophthalmological Surveillance Unit; ECCE, extracapsular cataract extraction; IOP, intraocular pressure; LA, local anaesthesia; PC, posterior capsule; $\mathrm{SCH}$, suprachoroidal haemorrhage 
effect of SCH. In the remaining 22 cases, PC rupture preceded $\mathrm{SCH}$; $\mathrm{SCH}$ only became apparent during anterior vitrectomy or wound closure. Posterior capsule rupture was therefore examined as a potential risk factor in these cases.

\section{Statistical analysis}

Potential risk factors were individually compared between cases and controls in univariate analyses, using $\chi^{2}$ or Fisher's exact tests for categorical variables, and Mann-Whitney tests for quantitative variables.

Logistic regression analyses were performed to test for independence among risk factors that were significant at the $\mathrm{p}<0.05$ level in the univariate analysis. To maximise the degrees of freedom, variables with $>15 \%$ of missing data and those present in small numbers $(<4 \%)$ were excluded. ${ }^{6}$ The final logistic regression model was constructed using a combination of forward stepwise inclusion and backward elimination paradigm.

\section{RESULTS}

\section{Univariate analysis}

Tables 1 and 2 give descriptive statistics of some of the categorical and continuous variables. A history of systemic hypertension, ischaemic heart disease, respiratory disease, taking aspirin, and warfarin were not significant risk factors for SCH. General anaesthesia $(p=0.012)$ and peribulbar LA $(\mathrm{p}<0.001)$ are protective.

\section{Multivariate analysis}

Significant risk factors in univariate analysis $(\mathrm{p}<0.05)$ were included in the regression model, with the exception of:

- Risk factors with >15\% missing data (diastolic BP, hyperlipidaemia and anaemia)

- Risk factors that occurred in small numbers $(<4 \%)$ (general anaesthesia, bleeding diathesis, past ocular surgery, peripheral vascular disease).

The regression model was constructed using complete data available for 79 cases and 352 controls. Independently significant risk factors for SCH are shown in table 3.

The cumulative effect of risk factors in table 3 can be explored by comparing two hypothetical patients. Patient A is a glaucomatous 90 year old taking cardiovascular medication, with preoperative IOP of $25 \mathrm{~mm} \mathrm{Hg}$ who does not have orbital compression before surgery, which is subsequently complicated by PC rupture and conversion to ECCE. Patient B is a 70 year old cardiovascular medication free patient without glaucoma, with a preoperative IOP of $15 \mathrm{~mm} \mathrm{Hg}$, who has orbital compression prior to uncomplicated phacoemulsification. According to the regression model, patient A has 158 times the risk of a preoperative SCH compared with patient B.

\section{DISCUSSION}

This study, the largest prospective series on SCH complicating cataract surgery to date, uses the case control methodology ${ }^{6} 7$

Table 1 Univariate analysis of some categorical variables in cases and controls

\begin{tabular}{|c|c|c|c|c|c|}
\hline \multirow[b]{2}{*}{ Categorical variables } & \multicolumn{2}{|c|}{ Cases } & \multicolumn{2}{|c|}{ Controls } & \multirow[b]{2}{*}{ p Value } \\
\hline & Total & No of positive (\%) & Total & $\begin{array}{l}\text { No of positive } \\
(\%)\end{array}$ & \\
\hline \multicolumn{6}{|l|}{ Systemic } \\
\hline Cardiovascular drugs* & 108 & $65(58.3)$ & 449 & $128(28.5)$ & $<0.001$ \\
\hline Hyperlipidaemia & 56 & $10(17.9)$ & 448 & $28(6.3)$ & 0.005 \\
\hline Anaemia & 87 & $7(8.8)$ & 449 & $13(2.9)$ & 0.013 \\
\hline Peripheral vascular disease & e 98 & $7(7.1)$ & 448 & $9(2)$ & 0.014 \\
\hline Diabetes & 108 & $5(4.6)$ & 449 & $55(12.2)$ & 0.02 \\
\hline Female sex & 108 & $80(74)$ & 449 & $279(62)$ & 0.02 \\
\hline Bleeding diatheses & 101 & $3(3.1)$ & 447 & $2(0.4)$ & 0.046 \\
\hline Hypertension & 106 & $52(49.1)$ & 449 & $186(41.4)$ & $>0.1$ \\
\hline Heart Failure & 101 & $4(4.0)$ & 449 & $25(5.6)$ & $>0.1$ \\
\hline IHD & 101 & $12(11.9)$ & 449 & $50(11.1)$ & $>0.1$ \\
\hline CVA & 100 & $7(7.0)$ & 449 & $35(7.8)$ & $>0.1$ \\
\hline Respiratory disease & 105 & 13 (12.4) & 448 & $68(15.2)$ & $>0.1$ \\
\hline Aspirin & 104 & $21(20.2)$ & 449 & $109(24.3)$ & $>0.1$ \\
\hline Warfarin & 105 & $6(5.7)$ & 449 & $15(3.3)$ & $>0.1$ \\
\hline \multicolumn{6}{|l|}{ Ophthalmic } \\
\hline Glaucoma & 108 & $35(32.4)$ & 449 & $29(6.5)$ & $<0.001$ \\
\hline Past ocular surgery & 107 & $6(5.6)$ & 448 & $6(1.3)$ & 0.015 \\
\hline \multicolumn{6}{|l|}{ Operative } \\
\hline Procedure & 109 & & 449 & & \\
\hline Phacoemulsification & & $83(76.2)$ & & $415(92.4)$ & $>0.1$ \\
\hline ECCE & & $12(11.0)$ & & $26(5.8)$ & 0.038 \\
\hline Conversiont & & $14(12.8)$ & & $8(1.8)$ & $<0.001$ \\
\hline LA types $\ddagger$ & 105 & & 399 & & \\
\hline Peribulbar & & $47(44.8)$ & & $284(71.2)$ & $<0.001$ \\
\hline Sub-Tenon's & & $45(42.9)$ & & $83(20.8)$ & $<0.001$ \\
\hline Topical & & $11(10.5)$ & & $13(3.3)$ & $<0.001$ \\
\hline Orbital compression & 93 & $49(52.7)$ & 389 & 306 (78.7) & $<0.001$ \\
\hline $\mathrm{PC}$ rupture before $\mathrm{SCH}$ & 108 & $22(20.4)$ & 435 & $20(4.6)$ & $<0.001$ \\
\hline GA & 108 & $3(2.8)$ & 447 & $47(10.5)$ & 0.012 \\
\hline Consultant surgeon & 108 & $76(70.4)$ & 448 & $257(57.4)$ & 0.013 \\
\hline Right eye & 108 & $52(48.1)$ & 449 & $254(56.6)$ & $>0.1$ \\
\hline Scleral section & 108 & $10(9.3)$ & 448 & $36(8.0)$ & $>0.1$ \\
\hline \multicolumn{6}{|c|}{$\begin{array}{l}\text { *Beta blockers, diuretics, calcium channel antagonists, cardiac glycosides, angiotensin converting enzyme } \\
\text { inhibitors, angiotensin receptor blockers, alpha blockers, antiarrhythmic agents. } \\
\text { †Conversion: phacoemulsification conversion to ECCE. } \\
\text { tData not shown for retrobulbar LA and intracameral LA due to small numbers. } \\
\text { IHD, ischaemic heart disease; CVA, cerebral vascular accident; ECCE, extracapsular cataract extraction; LA, local } \\
\text { anaesthesia; PC, posterior capsule; SCH, suprachoroidal haemorrhage; GA, general anaesthesia; IOP, intraocular } \\
\text { pressure; BP, blood pressure. }\end{array}$} \\
\hline
\end{tabular}


Table 2 Univariate analysis of some continuous variables in cases and controls

\begin{tabular}{|c|c|c|c|c|c|c|c|}
\hline \multirow[b]{2}{*}{ Continous variables } & \multicolumn{3}{|l|}{ Cases } & \multicolumn{3}{|l|}{ Controls } & \multirow[b]{2}{*}{ p Value } \\
\hline & Number & Median & Range & Number & Median & Range & \\
\hline Age & 106 & 81.5 & $54-98$ & 447 & 77.0 & $20-96$ & $<0.001$ \\
\hline Preop. IOP $(\mathrm{mm} \mathrm{Hg})$ & 105 & 18 & $10-41$ & 445 & 16 & $7-38$ & $<0.001$ \\
\hline $\begin{array}{l}\text { Preop. diastolic BP } \\
(\mathrm{mm} \mathrm{Hg})\end{array}$ & 87 & 85 & 68-117 & 316 & 82 & $50-130$ & 0.047 \\
\hline $\begin{array}{l}\text { Preop. systolic BP } \\
(\mathrm{mm} \mathrm{Hg})\end{array}$ & 87 & 160 & $105-222$ & 316 & 153 & $90-219$ & 0.08 \\
\hline Axial length $(\mathrm{mm})$ & 104 & 23.1 & $19.7-29.8$ & 444 & 23.1 & $20.3-34.5$ & $>0.1$ \\
\hline
\end{tabular}

to identify risk factors for SCH. The main limitation is a possible case selection bias, which depended entirely on voluntary reporting. However, we have evidence to suggest a high level of case ascertainment. ${ }^{8}$ Compared with previous institutional ${ }^{12}$ or personal $^{34}$ series, it benefits from case sampling from a much wider source.

Any risk factors identified have to be considered in the context of the pathophysiology of SCH. Available information points to ocular hypotony as a major precipitating factor. ${ }^{9}{ }^{10}$ Unopposed by normal IOP during hypotony, increased choroidal transmural venous pressure results in a serous effusion within the suprachoroidal space. As this accumulates, the short and long posterior ciliary vessels that traverse the suprachoroidal space become stretched, and SCH occurs when these vessels rupture from excessive stretching.

Older age is associated with sclerosis and increased fragility of choroidal vessels, and has previously been implicated as a risk factor. ${ }^{13}$ Taking cardiovascular medication appears to be a better predictor of SCH than hypertension and elevated preoperative systolic BP. ${ }^{11}{ }^{12}$ Although higher preoperative diastolic BP was positive $(p=0.047)$ in the univariate analysis, it was not entered into the regression models due to incomplete data.

The association between glaucoma and SCH has also been implicated..$^{1}{ }^{12} 13$ Manschot postulated that glaucoma promotes "necrosis" of the posterior ciliary arteries. ${ }^{12}$ Others suggested chronically elevated IOP somehow weakens the integrity of the ciliary vessels by impeding the transmural flow of nutrients to the ciliary vessel wall. ${ }^{313}$

Orbital compression may be protective against SCH through its influence on IOP. Following peribulbar anaesthesia, IOP is either increased ${ }^{14}$ or remains unchanged, ${ }^{15}{ }^{16}$ while sub-Tenon's is known to cause little or no increase in IOP. ${ }^{17}{ }^{18}$ However, IOP is uniformly reduced following orbital compression $^{14-1619}$ regardless of the LA technique. In the regression model, orbital compression is selected while LA type is rejected as a significant risk factor, elegantly

Table 3 Independently significant risk factors for $\mathrm{SCH}$ in the multivariate logistic regression model $(n=431)$

\begin{tabular}{lllr}
\hline Variable & Odds ratio & $95 \% \mathrm{Cl}$ & p Value \\
\hline Age & 1.06 & $1.03-1.10$ & $<0.001$ \\
Cardiovascular drugs & 1.66 & $1.27-2.16$ & $<0.001$ \\
Glaucoma & 5.9 & $2.9-11.8$ & $<0.001$ \\
IOP & 1.09 & $1.02-1.17$ & 0.015 \\
Orbital compression & 0.31 & $0.18-0.55$ & $<0.001$ \\
PC rupture before SCH & 3.9 & $1.7-8.9$ & 0.001 \\
ECCE & 2.08 & $0.88-4.94$ & 0.096 \\
Conversion* & 6.4 & $2.2-18.9$ & 0.001 \\
\hline
\end{tabular}

*Conversion: phacoemulsification conversion to ECCE. $\mathrm{Cl}$, confidence interval; $\mathrm{PC}$, posterior capsule; $\mathrm{SCH}$, suprachoroidal haemorrhage; ECCE, extracapsular cataract extraction; IOP, intraocular pressure. demonstrating that the association of LA type with SCH is likely due to the different rate of orbital compression (peribulbar $80.9 \%$, sub-Tenons $24.4 \%$, topical $9.1 \%$ ). Despite concerns of possible trauma to ciliary vessels with orbital compression, ${ }^{120}$ our results show that it has a protective effect.

Posterior capsule rupture, elective ECCE, and phacoemulsification conversion-all significant operative risk factors for $\mathrm{SCH}$-have in common a higher risk of intraoperative hypotony compared with uncomplicated phacoemulsification. Posterior capsule rupture is often accompanied by vitreous loss, and anterior vitrectomy can mean more protracted surgery with increased risk of hypotony. The larger section of an elective or converted ECCE is also more predisposed to hypotony.

Based on the results of our study, certain recommendations regarding the modifiable risk factors can be made to minimise the risk of $\mathrm{SCH}$ in cataract surgery. Elevated preoperative IOP should be optimally controlled preoperatively. Further softening of the eye by orbital compression is also recommended, regardless of the LA technique. Large incision cataract surgery should be avoided if possible. In cases where ECCE is unavoidable, or when phacoemulsification is converted to ECCE, additional care should be taken to avoid hypotony (for example, pre-placed sutures to allow for more effective reforming of the anterior chamber following nuclear extraction). During anterior vitrectomy, the risk of hypotony can be reduced by use of a "two port" anterior vitrectomy system, with an independent infusion port to optimally maintain the IOP. ${ }^{21}$

In conclusion, this study confirms that multiple risk factors are involved in the pathogenesis of $\mathrm{SCH}$ during cataract surgery. We suggest that older patients with a history of glaucoma, and those taking cardiovascular medication(s) be identified as being at risk of SCH. Attention to the various modifiable risk factors is recommended in all patients, but especially in those at risk. Controlling the preoperative IOP, applying orbital compression following LA, performing small incision cataract surgery, and scrupulously avoiding peroperative hypotony may all help to reduce the risk of $\mathrm{SCH}$ in cataract surgery.

\section{ACKNOWLEDGEMENTS}

This project was made possible by the data contribution of over 100 ophthalmologists throughout the UK, which the authors wish to gratefully acknowledge. In addition, the authors would also like to thank the "control centres" for providing data on the controls: Wycombe General Hospital, Central Middlesex Hospital, St George's Hospital, Bristol Eye Hospital, Musgrove Park Hospital-Taunton, Royal United Hospital-Bath, Southport and Formby District General Hospital, Furness General Hospital, West Cumberland Hospital, Leeds General Infirmary, Alexandra Hospital, Reddich, Coventry and Warwickshire Hospital, and Princess Alexandra Eye Pavilion, Edinburgh.

The authors are very grateful for the support of the British Ophthalmological Surveillance Unit, and for the contribution of $\mathrm{Mr}$ Barny Foot in the preparation of the manuscript. We wish to thank 
Mrs Persis Ladwa for her administrative help, Dr Tineke Freni for her constructive advice, and Ellen Farleigh, James Harvey, Hugh James, and Clare Maley for data entry.

This project was supported by the Torbay Medical Research Fund, and the British Council for Prevention of Blindness, for which we are grateful.

\section{Authors' affiliations}

R Ling, M Cole, C James, Department of Ophthalmology, Torbay Hospital, Torquay, UK

S Kamalarajah, Eye and Ear Clinic, Royal Victoria Hospital, Belfast, UK S Shaw, Department of Mathematics \& Statistics, University of Plymouth, UK

Correspondence to: Mr R Ling, Consultant Ophthalmologist, West of England Eye Unit, Royal Devon \& Exeter Hospital (Wonford), Barrack Road, Exeter EX2 5DW, UK; roland.ling@rdehc-tr.swest.nhs.uk

Accepted for publication 1 September 2003

\section{REFERENCES}

1 Speaker MG, Guerriero PN, Met JA, et al. A case-control study of risk factors for intraoperative suprachoroidal expulsive hemorrhage. Ophthalmology 1991;98:202-10.

2 Beatty S, Lotery A, Kent D, et al. Acute intraoperative suprachoroidal haemorrhage in ocular surgery. Eye 1998;12:815-20.

3 Davison JA. Acute intraoperative suprachoroidal hemorrhage in

extracapsular cataract surgery. J Cat Refract Surg 1986;12:606-22.

4 Arnold PN. Study of acute intraoperative suprachoroidal hemorrhage. $J$ Cataract Refract Surg 1992;18:489-494.

5 Foot B, Stanford M, Rahi J, et al. The British Ophthalmological Surveillance Unit: an evaluation of the first 3 years. Eye 2002;16:1-7.
6 Hosmer DW, Lemeshow S. Applied logistic regression, 2nd ed. New York: Wiley, 2000

7 Wilson MR, Hertzmark E, Walker AM, et al. A case-control study of risk factors in open angle glaucoma. Arch Ophthalmol 1987;105:1066-71.

8 Ling R, Cole M, James C, et al. Suprachoroidal haemorrhage complicating cataract surgery in the UK: epidemiology, clinical features, management, and outcomes. Br J Ophthalmol 2004;88:478-80.

9 Wolter JR, Garfinkel RA. Ciliochoroidal effusion as precursor of suprachoroidal hemorrhage: a pathologic study. Ophthalmic Surg 1988;19:344-9.

10 Beyer CF, Peyman GA, Hill JM. Expulsive choroidal hemorrhage in rabbits: a histopathologic study. Arch Ophthalmol 1989:107:1648-53.

11 Hoffman P, Pollack A. Oliver M. Limited choroidal hemorrhage associated with intracapsular cataract extraction. Arch Ophthalmol 1984;102:1761-5.

12 Manschot WA. The pathology of expulsive hemorrhage. Am J Ophthalmol 1955:40:15-24.

13 Taylor DM. Expulsive hemorrhage. Am J Ophthalmol 1974;78:961-6.

14 Bowman R, Liu C, Sarkies N. Intraocular pressure changes after peribulbar injections with and without ocular compression. Br J Ophthalmol 1996:80:394-7.

15 Ling R, Beigi $B, Q u i n n A Q$, et al. Effect of Honan balloon compression on peribulbar anaesthesia adequacy in cataract surgery. I Cataract Refract Surg 2002:28:113-17.

16 Morgan JE, Chandna A. Intraocular pressure after peribulbar anaesthesia: is the Honan balloon necessary? Br J Ophthalmol 1995;79:46-9.

17 Pianka P, Weintraub-Padova H, Lazar M, et al. Effect of sub-Tenon's and peribulbar anesthesia on intraocular pressure and ocular pulse amplitude. $J$ Cataract Refract Surg 2001;27:1221-6.

18 Alwitry A, Koshy Z, Browning AC, et al. The effect of sub-Tenon's anaesthesia on intraocular pressure. Eye 2001;15:733-5.

19 McDonnell PJ, Quigley HA, Maumenee AE, et al. The Honan intraocular pressure reducer. Arch Ophthalmol 1985;103:422-5.

20 Spaeth GL. Commentary. Ophthalmol 1991;98:210.

21 Blumenthal M, Grinbaum A, Assia El. Preventing expulsive hemorrhage using an anterior chamber maintainer to eliminate hypotony. J Cataract Refract Surg 1997;23:476-9. 